\title{
ESTIMATION AND IDENTIFICATION OF STRUCTURAL PARAMETERS IN THE PRESENCE OF MULTIPLE EQUILIBRIA
}

\author{
Russell W. Cooper \\ Working Paper 8941 \\ http://www.nber.org/papers/w8941 \\ NATIONAL BUREAU OF ECONOMIC RESEARCH \\ 1050 Massachusetts Avenue \\ Cambridge, MA 02138 \\ May 2002
}

The views expressed herein are those of the author and not necessarily those of the National Bureau of Economic Research.

(C) 2002 by Russell W. Cooper. All rights reserved. Short sections of text, not to exceed two paragraphs, may be quoted without explicit permission provided that full credit, including $\mathbb{C}$ notice, is given to the source. 
Estimation and Identification of Structural Parameters in the Presence of Multiple Equilibria Russell W. Cooper

NBER Working Paper No. 8941

May 2002

JEL No. E1, E3, E6, J7, Z13

\begin{abstract}
This paper studies quantitative implications of model economies that exhibit multiple equilibria. The goal is to assess two interrelated issues. First, do economies with multiple equilibria have falsifiable predictions? Second, is identification possible in economies that exhibit multiple equilibria? Put differently, are these economies observationally equivalent to economies with unique equilibria? We raise these questions within a general framework and then study a series of examples to determine how the existing literature has addressed them.
\end{abstract}

\author{
Russell W. Cooper \\ Department of Economics \\ Boston University \\ 270 Bay State Road \\ Boston, MA 02215 \\ and NBER \\ rcooper@bu.edu
}




\title{
Estimation and Identification of Structural Parameters in the Presence of Multiple Equilibria
}

\author{
Russell W. Cooper* \\ Department of Economics, Boston University, Boston, MA. 02215, USA \\ rcooper@bu.edu
}

May 6, 2002

\begin{abstract}
This paper studies quantitative implications of model economies that exhibit multiple equilibria. The goal is to assess two interrelated issues. First, do economies with multiple equilibria have falsifiable predictions? Second, is identification possible in economies that exhibit multiple equilibria? Put differently, are these economies observationally equivalent to economies with unique equilibria? We raise these questions within a general framework and then study a series of examples to determine how the existing literature has addressed them.
\end{abstract}

\section{Overview}

There are now a plethora of examples of economies which exhibit multiple equilibria. These examples exist (even thrive) in almost all aspects of economics: business cycle theory, open economy macroeconomics, game theory, growth theory, income distribution, industrial organization, labor, etc. The popularity of this approach indicates that the multiple equilibrium perspective provides insights into a variety of economic observations. In some cases, these models have been used, often informally, as a basis for policy interventions. ${ }^{1}$

But, to many economists, model economies that exhibit multiple equilibria are viewed as poorly suited for economic analysis. In particular, it is sometimes claimed that these models have no testable implications and thus cannot be falsified. ${ }^{2}$ Further, due to the multiplicity they are supposedly not useful for comparative static exercises that underly policy analysis: with multiple equilibria, it is not clear how policy variations will effect outcomes, absent a selection mechanism.

\footnotetext{
${ }^{*}$ I am grateful to the NSF for financial support and the Bernard Caillaud and Hubert Kempf for the opportunity to present these ideas at the September 2001 ADRES Conference. I thank my discussants, Stéphane Grégoir and Franck Portier, as well as seminar participants for their insightful comments and questions. I am also grateful to Victor Aguirregabiria, Simon Gilchrist and Andrea Moro for many discussions on this topic.

${ }^{1}$ Included here would be explicit policies such as deposit insurance, government provided guarantees, numerous discussions of currency crises and debates about the nature of aggregate fluctuations that rely, in part, on fluctuations in confidence.

${ }^{2}$ See, for example the discussion in Woodford (1987) and the comments in Aiyagari (1995) on these points.
} 
For those economists who think that allowing multiplicity is a useful (essential) way to confront reality, these arguments provide a challenging critique. For those who want to dismiss the multiple equilibrium perspective these are powerful arguments. Either way, we ought to evaluate them. That is the purpose of this paper.

More precisely, the goal is to make progress on answering two questions that bear on the ability of models with multiple equilibria to confront data:

- Can a model with multiple equilibria be rejected?

- Can the parameters of a model with multiple equilibria be identified?

While clearly related, these are intended to be two different questions. The first takes as given a model economy with multiple equilibria and asks whether there are observations that could falsify the model. The second question considers a larger set of economies (that includes the model with multiple equilibria) and asks whether the parameters of the model can be identified.

The approach we take is to examine a series of examples from various branches of economics. ${ }^{3}$ At one level, these examples indicate the usefulness of models of multiple equilibria in an informal way: the researcher has some observations which motivate the consideration of a model with multiple equilibria. But, our goal is to go further by examining how these examples of multiple equilibria have been used to confront data. Thus we are forced to address the issue of identification in models economies with multiple equilibria. ${ }^{4}$

The first part of this essay provides a general framework for the discussion of these issues. ${ }^{5}$ In addition, some abstract examples, drawing on Jovanovic (1989) and Bryant (1983), are presented to fix ideas. In the second part of the essay, we examine a variety of applications to better understand how researchers are coping with identification issues when evaluating models of multiple equilibria. ${ }^{6}$

\section{Abstract Formulation of the Problem}

While many applications studied below are dynamic, the basic themes of this essay can be adequately conveyed in a static formulation. We use this structure to discuss multiple equilibria and then estimation. This section concludes with some examples.

\subsection{Theory}

Following Cooper and John (1988), consider a game played by a large number of agents. Each agent chooses an action e from a compact, convex subset of $\Re$. The payoff of the agent is given by $\sigma(e, E ; X, \Theta)$ where $E$ is,

\footnotetext{
${ }^{3}$ Of course, theorems would be preferred but we start with examples to explore the themes.

${ }^{4}$ Identification is vital for the design of welfare improving interventions. However, in some cases, we may be interested in the presence of multiple equilibria alone.

${ }^{5}$ This structure follows Cooper and John (1988) and focuses on strategic complementarities as a basis for multiple symmetric Nash equilibria. Of course, multiple equilibria may arise in other games in which there are strategic substitutes, though these are not symmetric equilibria. Further, it is understood the multiple equilibria may also arise in fairly standard general equilibrium models as well as dynamic economies, such as overlapping generations models.

${ }^{6}$ Of course we have chosen a select set of examples for this purpose. There are many more in the literature.
} 
say, the average of the actions chosen by other players in the game, $X$ is the realized value of an exogenous variable that is common to all agents and $\Theta$ is a vector of parameters characterizing the payoffs of the agent. Assume that $\sigma(e, E ; X, \Theta)$ is continuously differentiable and strictly concave in $e$. The agent's optimization problem generates an unique optimal response given by $e=\phi(E ; X, \Theta)$, a reaction function. This is shown in Figure 1.

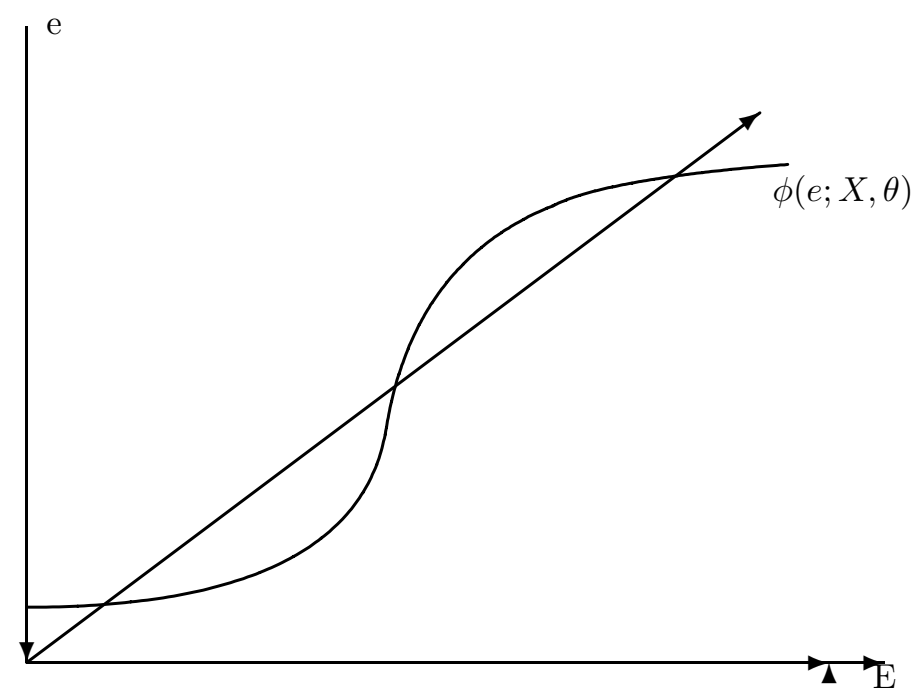

Figure 1: Policy Function

Given that all agents have identical payoffs, we focus on the set of symmetric Nash equilibria defined by

$$
\xi(X, \Theta)=\{e \mid e=\phi(e ; X, \Theta)\}
$$

Assume that the set of Nash equilibria $\xi(X, \Theta)$ is non-empty for all values of $(X, \Theta){ }^{7}$

Note though that the equilibrium need not be unique. As argued in Cooper and John (1988), a necessary condition for multiple symmetric Nash equilibria is strategic complementarity: $e=\phi(E ; X, \Theta)$ is increasing in $E .{ }^{8}$ For the purposes of our discussion here, the issue is not conditions for multiplicity per se but rather the implications of multiplicity for the estimation of the parameter vector $\Theta$ and for evaluating how well the model fits observations.

This quantitative analysis requires the specification of a mapping from the set of equilibria into a time series (or panel) of outcomes. One common approach is to assume the exists of a random variable (a sunspot), observed to all agents, selects from the set of Nash equilibria. This is often viewed as extrinsic uncertainty in that this random variable does not influence anything fundamental to the problem, it simply influences beliefs.

\footnotetext{
${ }^{7}$ See Cooper (1999) for more details on conditions such that this set is nonempty.

${ }^{8} \mathrm{As}$ is understood, these strategic complementarities also can magnify and propagate shocks.
} 
Let $\Pi(X, \Theta)$ represent the set of probability distributions over the set of equilibria, $\xi(X, \Theta)$. A sunspot process is given by a $\pi \in \Pi$ such that $\pi$ specifies the likelihood of observing each equilibrium in $\xi(X, \Theta) .{ }^{9}$ Of course, this sunspots approach brings a lot of "coordination" to a problem of coordination and is thus somewhat unappealing. Instead, one might imagine a more elaborate process by which individual agents' beliefs dictate their actions and those beliefs then evolve as a function of history. Alternatively, as discussed in Jovanovic (1989), one might look more generally at the restrictions imposed by the set of Nash equilibria without any selection from that set.

\subsection{Estimation}

Suppose that a data set is generated by this "economy" consisting of $D=\left(e_{t}, X_{t}\right)$ for $t=1,2, \ldots T . .{ }^{10}$ The problem for the applied economist is to use $Z$ to infer something about $\Theta$ and about the sunspot process. Denote by $\Theta^{+}$the expanded parameter set that includes both the primitive parameters $\Theta$ and those that describe the sunspot process. ${ }^{11}$ We discuss two approaches: method of moments and maximum likelihood.

\subsubsection{Method of Moments}

Let $M_{T}$ be a set of moments calculated from this data set. ${ }^{12}$ Further, let $M(\Theta)$ be a mapping from the parameters of the coordination game to a set of moments calculated from a simulated data set that, like $D$, includes observations on economy efforts and the exogenous variables. We denote this data set, contingent on $\Theta^{+}$as $\left\{D\left(\Theta^{+}\right)\right\}$. To create this simulated data set, imagine generating a sequence of exogenous variables, $X$, and, for a given $\Theta^{+}$, generating a sequence of equilibrium actions, perhaps through a sunspot process to select from the set of Nash equilibrium. Calculating a set of moments from this simulated data set creates $M\left(\Theta^{+}\right)$.

Then one approach to estimation solves:

$$
\min _{\Theta^{+}}\left(M_{T}-M\left(\Theta^{+}\right)\right)^{\prime} W\left(M_{T}-M\left(\Theta^{+}\right)\right)
$$

where $W$ is a square weighting matrix and $M_{T}-M\left(\Theta^{+}\right)$is the difference between the actual and the simulated moments. When the model is exactly identified (the number of elements in $\theta^{+}$equals the number of moments), then $W$ is immaterial. But, if the model is overidentified (the number of elements in $\Theta^{+}$is less than the number of moments), then the choice of $W$ is important for efficiency.

\footnotetext{
${ }^{9}$ The sunspot process need not put positive probability on all equilibria. These restrictions may be imposed by the researcher a priori.

${ }^{10}$ In the applications, we will discuss further the nature of observables to the researchers versus the private agents. As all shocks are common and all agents are identical, there is no additional information contained in a panel as long as the agent's choice are part of a symmetric Nash equilibrium. We assume for now that this is indeed the case. Carlsson and van Damme (1993) argue that in economies with heterogeneous agents due to incomplete information, there may be a natural selection from the set of equilibria.

${ }^{11}$ If for example, the sunspot process follows a first-order Markov process, then $\Theta^{+}$would include the elements of the transition matrix characterizing the sunspot process.

${ }^{12}$ At this point we are not that precise about which moments are here. Details on that important issue follow below.
} 
This is a good point to be a bit more precise about the implications of identification and multiplicity of equilibria. Suppose, that $M(\Theta)$ is a one-to-one function: for each value of $\Theta$ the model generates a unique set of moments and for each value of the moments, there is only one value of $\Theta$ that could generate that value. ${ }^{13}$ In that case, there is clearly only one value of $\Theta$ that could generate the observed moments. Clearly, the minimization problem in (1) produces a consistent estimate of $\Theta .{ }^{14}$ This case is illustrated in Figure $2 .{ }^{15}$

Figure 2: Just Identification

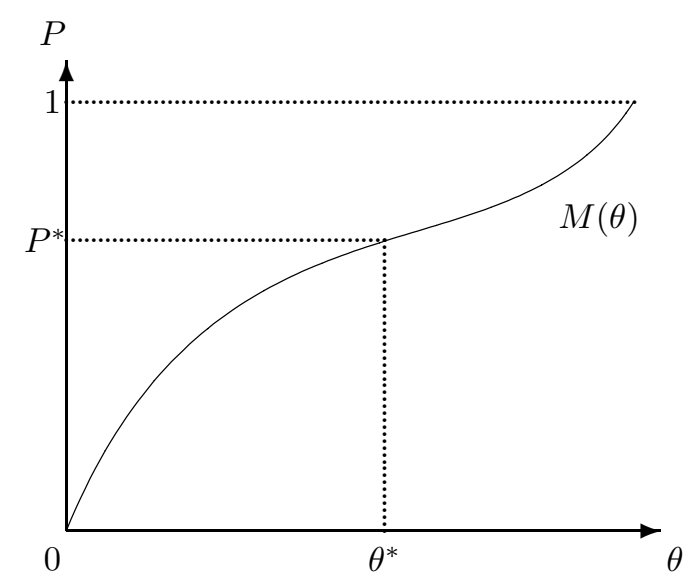

The problem of identification arises when there are multiple values of $\Theta$ that can match the observed moments. Note that here the model is identified locally (there is not a problem of local flats) but there is no global identification. In other cases (examples are given below), the identification problem may be local. This is shown in Figure 3.

In the case of multiple equilibria: for a given $\Theta$, there are multiple moments that can be generated. ${ }^{16}$ So, $M(\Theta)$ is not a function. This is logically distinct from the standard identification problem. But, it does turn out, that some models may exhibit both multiple equilibria and also have identification problems. This case is shown in Figure 4.

As we shall see, in some cases when the sunspot variables are added to $\Theta$ to create $\Theta^{+}$, an identification problem arises. Importantly, this depends, among other things, on the moments selected. Though the underlying sunspot may not be observed by the econometrician, variations in the transition matrix may have observable implications and thus the sunspot process may be identified. Still, a key issue in the identification is the discrimination between two sources of variations in observable economic variables: sunspots and fundamental shocks $(X) .{ }^{17}$

\footnotetext{
${ }^{13}$ Note that here we are excluding the sunspot variables from $\Theta$ as we are assuming there is a unique equilibrium.

${ }^{14}$ See the discussion and references in Gourieroux and Monfort (1996) and Hansen (1982).

${ }^{15}$ This figure and the others that follow are taken from joint work with Jérôme Adda.

${ }^{16}$ Note again that the domain here is $\theta$.

${ }^{17}$ This is an identification problem: can we jointly estimate the probability distributions of both fundamentals and sunspots?
} 
Figure 3: Non Identification
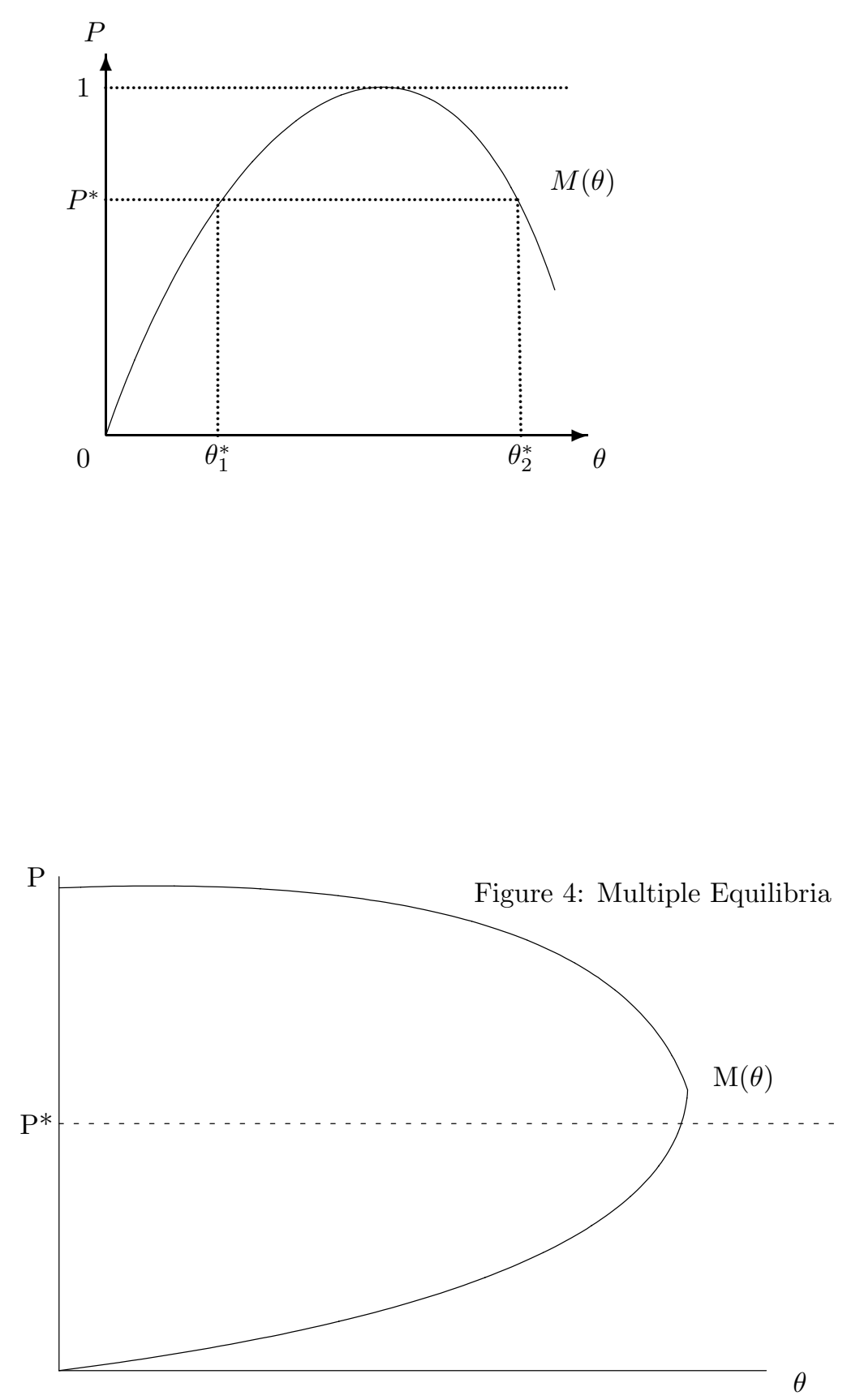


\subsubsection{Maximum Likelihood}

A second approach is to consider the estimation of parameters through a maximum likelihood procedure. ${ }^{18}$ In the usual case of a linear regression model, $y=X \beta+\varepsilon$, we make an assumption about the distribution of the errors. ${ }^{19}$ we then express the likelihood of the data as a function of the parameters $(\beta)$ of the model and proceed to the maximization of the likelihood function.

In the presence of multiple equilibria, maximizing a likelihood function is not as easy. ${ }^{20}$ First, economies that generate multiple equilibria are inherently nonlinear so that simple linear representations may be inadequate. Second, the sunspot variables reappear as a type of latent variable, observable to private agents but not to the researcher.

Suppose we have the data set $D$ and wish to write down the likelihood of an observation for a particular year, $\left(e_{t}, X_{t}\right){ }^{21}$ Partition $X_{t}$ into two components, $\left(X_{t}^{1}, X_{t}^{2}\right)$ and assume that while $X_{t}$ is observed by private agents, only $X_{t}^{1}$ is observable to the researcher. So, $X_{t}^{2}$ plays the role of the error term in the linear regression. Then, the likelihood of observing $\left(e_{t}, X_{t}^{1}\right)$ is:

$$
\mathcal{L}_{\Theta}\left(e_{t}, X_{t}^{1}\right)=q \mathcal{L}_{\Theta}^{L}\left(e_{t}, X_{t}^{1}\right)+(1-q) \mathcal{L}_{\Theta}^{H}\left(e_{t}, X_{t}^{1}\right)
$$

Here, $q$ represents the probability of the pessimistic sunspot (superscript $L$ ) variable and $(1-q)$ is the probability of optimism (superscript $H$ ). For each realization of the sunspot variable, there is a distinct piece of the likelihood function. ${ }^{22}$ Effectively, these branches of the likelihood function calculate the probability of observing $\left(e_{t}, X_{t}^{1}\right)$ conditional on the sunspot variable.

The likelihood function thus integrates over the two random variables not observed by the researcher. The first is the sunspot variable. The second is $X_{t}^{2}$, the part of $X_{t}$ that only agents observe. Thus, $\mathcal{L}_{\Theta}^{j}\left(e_{t}, X_{t}^{1}\right)$, for $j=H, L$ is the probability of $X_{t}^{2}$ taking a value so that we would observe $\left(e_{t}, X_{t}^{1}\right)$, given that the sunspot takes value $j$.

With this construction, the likelihood of the sample is then:

$$
\mathcal{L}_{\Theta}(D)=\prod_{t}\left(q \mathcal{L}_{\Theta}^{L}\left(e_{t}, X_{t}^{1}\right)+(1-q) \mathcal{L}_{\Theta}^{H}\left(e_{t}, X_{t}^{1}\right)\right)
$$

Maximization of this likelihood will lead to estimates of the underlying parameters of the payoff functions as well as to estimates of $q$. Essentially, the probability of the optimistic sunspot variable is determined by the likelihood function putting weight on each of two regimes (optimism and pessimism) so as to maximize the likelihood of observing $D$.

\footnotetext{
${ }^{18}$ I am grateful to Stéphane Grégoir, Simon Gilchrist and Paul Ruud for discussions on this material.

${ }^{19}$ Note that the method of moments does not need any such assumption.

${ }^{20}$ See Breshnahan and Reiss (1991) for a discussion of problems with maximum likelihood estimation in games with multiple equilibria and illustrations in an entry game.

${ }^{21}$ Once we have a likelihood for a single observation we can quickly generate a likelihood function for the entire sample given that the shocks are assumed to be iid. If they are not, then the likelihood is slightly more complicated.

${ }^{22}$ See Bisin and G.Topa (2002) for an alternative approach that puts all the weight in the likelihood function on the most likely equilibrium and thus does not utilize an explicit sunspot process.
} 


\section{$2.3 \quad$ Examples}

Here we illustrate these issues by building upon an example from Jovanovic (1989). Consider a game played by two agents, $i=1,2$. Each agent can choose to participate in an activity: $x_{i}=1$ indicates participation by agent $i$ and $x_{i}=0$ means no participation. If the agent chooses not to participate, then agent $i$ receives a payoff of zero: $\sigma_{i}=0$. If agent $i$ chooses to participate, then the payoff is given by:

$$
\sigma_{i}=\lambda x_{-i}-\mu
$$

where $x_{-i}$ is the action taken by the other agent. Here $\lambda$ is a parameter that we are seeking to estimate and $\mu$ is a random shock to the value of participation (assume that it is a common shock). Suppose that $\mu$ is uniformly distributed on $[0,1]$ so there are no parameters of this distribution to estimate. If $\lambda$ is positive, the model exhibits a strategic complementarity: the value of participating is higher if the other agent participates.

In fact, this complementarity is sufficiently strong that it is always an equilibrium for neither agent to participate. That is, if $x_{-i}=0$, then the best response of agent $i$ is to set $x_{i}=0$ as well regardless of the realized value of $\mu$.

But, for some values of $\mu$, there is also an equilibrium with participation. ${ }^{23}$ In particular, if $\lambda \geq \mu$, then $x_{1}=x_{2}=1$ is a Nash equilibrium. Still, even when $\lambda \geq \mu$, the equilibrium with $x_{1}=x_{2}=0$ exists. The equilibrium in $x_{1}=x_{2}=1$ clearly Pareto dominates the one with $x_{1}=x_{2}=0$. A coordination failure occurs when $\lambda \geq \mu$ and the equilibria with $x_{1}=x_{2}=0$ is observed. This is a form of social but not individual inefficiency.

Suppose that a researcher observes a time series of actions chosen by the two agents: $D=\left(x_{1 t}, x_{2 t}\right)$ for $t=1,2,3, \ldots T .{ }^{24}$ The goal of the researcher is to estimate the single parameter that governs the interaction between the agents, $\lambda$. Knowing $\lambda$ is important for determining if there are coordination problems and also in designing remedies. Can the researcher estimate $\lambda$ ?

Here the issue of equilibrium selection is important for identification. For example, suppose that agents in this economy are very pessimistic about the participation of the other agent. This pessimism leads them never to participate, regardless of the realization of $\mu$. In this Nash equilibrium, the time series of participation decisions will clearly not be informative about $\lambda$ : identification is not possible.

Alternatively, suppose that agents are optimistic so that in the event of multiple equilibria, agents participate. In this Nash equilibrium, $x_{1}=x_{2}=1$ will be observed whenever $\lambda \geq \mu$. The researcher will then observe a fraction of the time series in which agents participate and a fraction in which they do not. Given the assumption that the distribution of $\mu$ is uniform on $[0,1]$, the maximum likelihood estimate of $\lambda$ will equal the fraction of observations in which $x_{1}=x_{2}=1$.

Finally, suppose that if there are multiple equilibria $(\lambda \geq \mu)$, then agents will participate with probability $q$. Then observed participation rates yield an estimate of the product $q \lambda$ but not the individual terms. Nonetheless, in this case the researcher can determine whether a complementarity is present and thus whether

${ }^{23}$ There is also a mixed strategy equilibrium.

${ }^{24}$ So here the unobserved variable to the researcher but not to the private agents is $\mu$. 
there is a potential for coordination failure.

A second example draws upon the effort game presented in Bryant (1983). This example, with a continuum of symmetric Nash equilibria, illustrates the severity of the identification problem.

Suppose there are $I$ agents each setting a level of effort $\left(e_{i}\right)$ for $i=1,2, . . I$ in the interval $[0,1]$. Suppose that payoffs are given by:

$$
\sigma\left(e_{i}, e_{-i}\right)=a \min (e)-e_{i}
$$

where $e$ is the vector of effort levels by the I agents so that $\min (e)$ is the lowest effort level and $a$ is a parameter. If $a>1$, then there is a continuum of Nash equilibria indexed by the level of effort in $[0,1]$.

Of course, $e_{i}=1$ for all $i$ is the Pareto dominant equilibrium and thus any effort level short of this is a coordination failure.

The issue though is what this model allows us to identify from observations. Suppose that the researcher observes the average level of effort in this economy. In equilibrium, of course, this average level is the same as the equilibrium level. Absent a mechanism for selection, for a given value of $a>1$, any level of activity could be an equilibrium. Further, and more importantly for identification, the set of equilibria does not vary with the parameter $a$, as long as it exceeds unity. Thus this example illustrates a case where the moment predicted by a model may not even be locally unique: identification is not possible. Note though that this example is special in that the multiplicity is extreme: none of the equilibria are locally unique.

\section{Financial Intermediation and the Great Depression}

To illustrate these issues in an explicit example, we consider the economy explored in Cooper and Corbae (2001). One point of that exercise was to explore whether a model of multiple equilibria based upon a complementarity in the intermediation process could match certain features of the Great Depression. Here we focus on a simple parametric example taken from that paper.

The Cooper and Corbae (2001) overlapping-generations economy had a number of key ingredients:

- Heterogeneous households that lived for two periods; working in youth and consuming in both youth and old age

- two stores of value: money and "bank" deposits

- intermediaries with fixed costs of operation that are borne by the agents joining the intermediary

- firms that hire workers using funds borrowed from the intermediary

The key to the multiplicity of equilibrium is the endogenous market participation of the households. In one equilibrium, many households participate in intermediated activity thus increasing the returns to participation for all agents. In another equilibrium, many households hold money as a store of value. Consequently, the household who do join the intermediary bear relatively large fixed participation costs. Cooper and Corbae (2001) construct sunspot equilibria by randomizing across these two steady states. They are able to match some of the prominent features of the Great Depression in their parameterized economy. 


\subsection{Households}

The key to the model is the household's choice between holding money and joining the intermediary. Due to the presence of the fixed cost of operation, the return to joining the intermediary is an increasing function of the number of other agents in this coalition. Thus, as in Bryant (1987) and Weil (1987) there is a complementarity in the intermediation process. This complementarity can generate multiple equilibria: if many (few) of agents join the intermediary then the fixed costs borne by each household is relatively low (high) and thus (not) joining the intermediary is optimal.

A household of generation $t$ has preferences given by:

$$
\ln \left(c_{t}^{t}-\frac{n_{t}^{1+\gamma}}{1+\gamma}\right)+\beta E_{t} \ln \left(c_{t+1}^{t}\right)
$$

The labor supply decision of a household is simply

$$
n_{t}^{s}=w_{t}^{\frac{1}{\gamma}} .
$$

Note that this decision is independent of any intertemporal rates of return.

Given a real wage $\left(w_{t}\right)$ and real return on savings $\left(r_{t}\right)$, households choose to either hold money as a store of value or join an intermediary. If a household joins the intermediation coalition, they incur a fixed cost of $\tau$ and earn a marginal of $r_{t}$. The loan supply for this household is:

$$
l_{t}=\frac{\beta\left(\alpha-\tau_{t}\right)}{1+\beta}+\phi w_{t}^{\frac{1+\gamma}{\gamma}}
$$

where $\phi=\beta \gamma /[(1+\beta)(1+\gamma)]$ and $\alpha$ is the endowment. If a household holds money, then labor supply is again given by (7) and money demand is:

$$
m_{t}=\frac{\beta \alpha}{1+\beta}+\phi w_{t}^{\frac{1+\gamma}{\gamma}}
$$

Cooper and Corbae (2001) assume three types of households. Let type $i$ have endowment level $\alpha_{i}$ and let $A_{i}$ be the fraction of this type where $\alpha_{1}<\alpha_{2}<\alpha_{3}$. Let $H_{i}$ be the fraction of households with endowment level equal to or bigger than $\alpha_{i}$ and let $\mu\left(\alpha_{i}\right)$ be the mean endowment level for these households. Estimation of this simple income distribution is an important element in matching observations of the Great Depression.

\section{$3.2 \quad$ Firms}

Firms have access to a production technology that converts labor input in period $t$ into period $t+1$ output. Assume that the production function for a representative firm is $f(n)=\Psi n^{\xi}$ so that labor demand is

$$
n_{t}^{d}=\left[\frac{\xi \Psi}{w_{t}\left(1+r_{t}\right)}\right]^{\frac{1}{1-\xi}} .
$$

Note that labor demand depends on the interest rate as firms must borrow to finance their wage payments.

Cooper and Corbae (2001) assume two types of firms: type $j$ has fixed costs of production $k_{j}$ and $F_{j}$ is the fraction of firms with costs equal to or less than $k_{j}$, where $k_{1}<k_{2}$. As firms borrow to finance wage payments, their loan demand is $w_{t} n_{t}^{d}$. 


\subsection{Multiple Steady States and Sunspots}

A steady state equilibrium is characterized by a wage rate, an interest rate and a price level such that all agents (households and firms) behave optimally and all markets clear. For this example, there may be multiple steady states. The first is an optimistic equilibrium: type 1 households hold money while types 2 and 3 join the intermediary and all firms produce. The second is a pessimistic equilibrium: only type 3 households join the intermediary and only type 1 firms produce.

A stationary sunspot equilibrium indexes all the equilibrium prices and quantities by an extrinsic (sunspot) variable $(\theta)$ that determines which of the equilibria will arise in a given period. Cooper and Corbae (2001) argue that their model has two steady states. Given this, they construct a two-state sunspot equilibrium where the states are denoted optimism $\left(\theta=\theta_{o}\right)$ and pessimism $\left(\theta=\theta_{p}\right)$. Let the sunspot variable follow a first order Markov process and let $\Pi$ be a $2 x 2$ transition matrix for $\theta$. An element of $\Pi$ is given by:

$$
\Pi(i, j)=\operatorname{Pr}\left(\theta_{t+1}=\theta_{j} \mid \theta_{t}=\theta_{i}\right)
$$

We first discuss the set of steady states and then consider sunspots. For the economy outlined above, the equilibrium interest rates and wages (as functions of $\left(H_{\alpha^{*}}(\theta), F_{k^{*}}(\theta)\right)$ ) satisfy:

$$
1+r(\theta)=\Psi \xi\left(F_{k^{*}}(\theta)\right)^{1-\xi}\left[\frac{\left(\frac{1+\beta}{\beta}\right)\left(1-\phi H_{\alpha^{*}}(\theta)\right)}{\mu\left(\alpha^{*}(\theta)\right)-\Gamma}\right]^{\frac{\gamma+1-\xi}{1+\gamma}}
$$

and

$$
w(\theta)=\left[\frac{\Psi \xi\left(F_{k^{*}}(\theta)\right)^{1-\xi}}{1+r(\theta)}\right]^{\frac{\gamma}{1+\gamma-\xi}}
$$

From these expressions, an equilibrium with high household participation at intermediaries (optimism) will imply, from (12), low interest rates because the fraction of agents participating in loan activity $\left(H_{\alpha^{*}}\right)$ and the flow of loans $\left(\mu\left(\alpha^{*}\right)\right)$ will both be large. The effect on interest rates may be offset by higher firm participation $\left(F_{k^{*}}\right)$. From (13), wages will rise from the increased labor demand induced by the fall in interest rates and higher firm participation.

To guarantee that these conjectured asset market participation patterns are optimal requires a check on the participation decisions of the different households and firms. The expressions for interest rates and wages can be used to solve for the equilibrium choices of households and hence their expected lifetime utility. Recall that $\Delta(\theta)$ is the difference in utility between making loans and holding money. For this example,

$$
\Delta(\theta)=(1+\beta) \ln \left(1-\frac{\tau(\theta)}{\alpha+\omega(\theta)}\right)+\beta \ln (1+r(\theta))
$$

where

$$
\omega(\theta)=\left(\frac{\gamma}{1+\gamma}\right) w(\theta)^{\frac{1+\gamma}{\gamma}}
$$

and $\tau(\theta)=\Gamma / H_{\alpha^{*}}(\theta)$. From (14), we see the cutoff property for asset market participation: given $(w(\theta), r(\theta)$, $\tau(\theta))$ only agents with sufficiently large endowments will join the intermediary since the utility differential is increasing in $\alpha$.

The optimistic equilibrium with household types 2 and 3 joining the intermediary coalition arises if (14) is positive for endowment levels $\alpha_{2}$ and $\alpha_{3}$. This condition is evaluated with $\tau=\Gamma / H_{\alpha_{2}}$, using $H_{\alpha_{2}}$ and 
$\mu\left(\alpha_{2}\right)$ to determine interest rates and wages. In an optimistic equilibrium, we must also check that both firm types make positive profits. Similarly, the conditions for the pessimistic equilibrium can be checked as well.

If these conditions hold, then the economy displays multiple steady states, indexed by $\theta$. In effect, the multiple steady state correspond to a particular sunspot transition matrix, $\Pi$ with ones on the diagonal. But, there are generally other sunspot equilibria in the neighborhood of this extreme transition matrix. ${ }^{25}$ With the preferences assumed in the example, introducing sunspots will only influence the asset market participation decisions. In each of the steady states constructed above, all of the asset market participation decisions were strict inequalities. Therefore, a $\Pi$ matrix with elements near one on the diagonal will not change these decisions either.

Thus, it is easy to construct sunspot equilibria in the neighborhood of these two steady states. Let $\bar{\Pi}_{o o}$ and $\bar{\Pi}_{p p}$ denote two critical transition probabilities. Then by the arguments above (formalized in Cooper and Corbae (2001), there will exist sunspot equilibria for any $\Pi$ as long as $\Pi_{o o} \geq \bar{\Pi}_{o o}$ and $\Pi_{p p} \geq \bar{\Pi}_{p p}$.

Of course, these equilibria depend on the parameters of preferences, technology and the sunspot process. We turn to estimation now.

\subsection{Parameterization}

Let $\Theta^{+}$be the list of parameters that include:

- household parameters: $(\gamma, \beta)$

- wealth distribution

- firms parameters: $(\xi, \Psi)$

- distribution of firm's fixed costs

- fixed cost of intermediation: $\Gamma$

- the transition matrix for the sunspot variable, $\Pi$

The value of $\Theta^{+}$is chosen to minimize the distance between actual and simulated moments. This procedure will produce consistent estimates of $\Theta^{+}$. For their exercise, Cooper and Corbae (2001) only estimated the household income distribution and the verification costs as these were key to the multiplicity of equilibria. Of course, in principle, the estimation could have been broadened to estimate more parameters and more moments. ${ }^{26}$

Cooper and Corbae (2001) assume that the U.S. was in an optimistic equilibrium pre-1929 and then was in a pessimistic equilibrium over the 1930-33 period. This is necessary for the calibration since the only source of fluctuations is in the sunspot process. So, the estimation essentially chooses parameters to match

\footnotetext{
${ }^{25}$ As these models are built around strategic complementarity, the sunspots are in the neighborhood of the steady state. This contrasts with the conditions for sunspot equilibria in overlapping generations models, as in Azariadis (1981), as the connection to deterministic cycles, as in Azariadis and Guesnerie (1986), is not present.

${ }^{26}$ Further, the model could include more shocks and thus be more capable to match other moments.
} 
moments for the two steady states outlined above. ${ }^{27}$ In each case, the participation decisions are checked to guarantee that the multiplicity exists for the particular parameterization.

The moments chosen, under optimism and pessimism, were: real interest rates, currency/deposit ratios, deflation, output growth and velocity growth. These moments were selected for their salience in the ongoing discussion over the Great Depression. ${ }^{28}$

Tables 1 and 2 present the predictions of this economy, based upon the estimated (household income distribution) and calibrated (all others) parameter values given there. Cooper and Corbae compare steady states of the model against observations before (optimism) and during (pessimism) the Great Depression. ${ }^{29}$

Table 1

Steady State Values

\begin{tabular}{|c|c|c|}
\hline Variable & Optimism (model,data) & Pessimism (model,data) \\
\hline real interest rate $\%$ & $(8.6,7.4)$ & $(10.1,11.3)$ \\
\hline currency/deposit & $(0.079,0.086)$ & $(0.233,0.227)$ \\
\hline
\end{tabular}

Parameters: $\Gamma=0.0055,\left[k_{1}=0, k_{2}=0.06\right], F_{1}=0.95, \gamma=0.05,\left[\alpha_{1}=0.23, \alpha_{2}=0.25, \alpha_{3}=12\right]$,

$$
\left[A_{1}=0.36, A_{2}=0.55\right], \beta=0.9, \xi=0.9, \phi=1.11 \text {. }
$$

Table 2

\% Change: Optimism to Pessimism

\begin{tabular}{|c|c|}
\hline Variable & $($ model,data $)$ \\
\hline deflation & $(61,33)$ \\
\hline production & $(-11,-36)$ \\
\hline velocity & $(-65,-35)$ \\
\hline$\Delta$ loans/output & $(-12,-10)$ \\
\hline real wages & $(-0.6,-9)$ \\
\hline
\end{tabular}

From Table 1, the estimated model is able to match the currency/deposit ratio is higher in the pessimistic steady state, rising from 0.079 to 0.233 . It rose from 0.086 to 0.227 in U.S. data. The interest rate on loans rises from 8.6 to $10.1 \%$ while in U.S. data the real ex post return on short term government debt increased from $7.4 \%$ in 1929 to $11.3 \%$. The model is unable to match the movements in the loan/deposit ratio principally because our banks have no other assets to hold.

From Table 2:

- real output does not fall as much in the model as it does during the Great Depression. This reflects the absence of other factors such as real investment and inventory changes that may have magnified these effects.

- consequently, the model predicts too much deflation relative to observation

\footnotetext{
${ }^{27}$ As discussed below, this is sufficient to solve for a sunspot equilibrium.

${ }^{28}$ There were therefore 6 parameters estimated from 7 moments: the parameters were overidentified.

${ }^{29} \mathrm{~A}$ more recent version of that analysis introduces money growth into the model.
} 
- the model predicts a fall in loans relative to output but not in the loan/output ratio. This is consistent with observations

- given that labor supply is so flat, wages do not vary much.

One very interesting element in the example is that the fixed cost is only about $1 \%$ of deposits. Still, this small fixed cost can produce multiple equilibria due to the swing in the middle class whose asset holdings change across the two steady states.

In a sunspot equilibrium constructed by randomizing in the neighborhood of the two steady states, the movements of the variables across the states are given in Tables 1 and 2. Subject to there being a sunspot equilibrium, labor supply and savings decisions, given the special preferences assumed in this example, are independent of the sunspot process.

In fact, one can calculate how much persistence in each state is necessary to have a sunspot equilibrium. This is of interest since one suspects that pessimism is not close to a permanent state. For our example, we can support a sunspot equilibrium in which $\Pi_{o o} \geq 0.99$ while $\Pi_{p p} \geq 0.5$. Thus the pessimistic state need not be very persistent though the optimistic state is fairly persistent and thus switches to pessimism (such as a Great Depression episode) are relatively infrequent.

So, returning to the theme of this paper, is this an example of success in the "estimation" of a model built upon multiple equilibria? At one level, it certainly is since the mapping from parameters to observations (simple moments) allowed us to determine some of the key parameters of interest. Certainly there are parameters that we did not estimate but there is nothing in the procedure per se that limits the estimation in that way. The model, as noted above, is (informally) quite far from observation: we would reject the overidentified model. ${ }^{30}$

On the key issue of identification, note that in fact Cooper and Corbae were unable to go beyond restricting the sunspot process to an interval. Again, it appears that this is a consequence of the functional forms they choose in the analysis since real decisions on labor supply and savings appear to be insensitive to real returns. So, perhaps in a model with richer preferences, the sunspot process could be more precisely estimated from observations on consumption, employment and even asset prices.

A final point concerns a competing model. It might be possible to construct a model that has a unique equilibrium with fluctuations driven by real shocks to a banking technology. That model may appear to be observationally equivalent to the one constructed by Cooper and Corbae. Yet, at least informally, the response of the economy to confidence building measures by the Roosevelt Administration in the mid 1930s lends some additional support in favor of the sunspot perspective.

\section{Aggregate Fluctuations with Technological Complementarities}

Similar issues of identification arise in stochastic business cycle models in the presence of technological complementarities, see Bryant (1983), Benhabib and Farmer (1994), Baxter and King (1991) and Cooper

\footnotetext{
${ }^{30}$ Of course the model also does not allow any fluctuations conditional on a regime and hence is clearly at odds with observations on that basis. One might add other sources of fluctuations, such as TFP shocks.
} 
(1999). In these models, the production function for a representative firm includes some measure of aggregate production. The idea is to introduce a complementarity into the production process so that high levels of activity in other firms implies that a single firm is more productive as well. ${ }^{31}$ Let $y$ represent the output at a given firm, $Y$ be aggregate output, $k$ and $n$ the firm's input of capital and labor respectively. Consider a production function of:

$$
y=A k^{\alpha} n^{\phi} Y^{\gamma}
$$

where $A$ is a productivity shock that is common across producers. Here $\gamma$ parameterizes the interaction between producers. If $\gamma$ is positive, then there is a complementarity at work: as other agents produce more, the productivity of the individual agent increases as well. This production function can be imbedded into a stochastic growth model. Consider the problem of a representative household with access to a production technology given by (15). As all agents are identical and all shocks are common, the representative household will accumulate its own capital, supply its own labor and interact with other agents only due to the technological complementarity. Thus the household solves:

$$
\max E \sum_{t=0}^{\infty} \beta^{t} u\left(c_{t}, l_{t}\right)
$$

subject to:

$$
\begin{aligned}
l_{t}+n_{t} & =1 \\
c_{t}+i_{t} & =y_{t} \\
k_{t+1} & =k_{t}(1-\delta)+i_{t} \\
y_{t} & =A k_{t}^{\alpha} n_{t}^{1-\alpha} Y_{t}^{\gamma}
\end{aligned}
$$

In this optimization problem, the individual household takes $Y_{t}$ as given. In a symmetric equilibrium, $y_{t}=Y_{t}$. As in Baxter and King (1991), this equilibrium condition is neatly imposed through the firstorder conditions when the marginal products of labor and capital are calculated. From the set of first-order conditions, the symmetric equilibrium can be analyzed through by approximation around a steady state. ${ }^{32}$

The distinguishing feature of this economy from the traditional RBC model is the presence of the technological complementarity, $\gamma$. We focus our discussion on estimating this parameter either directly by estimation of (15) or by inferring it from the equilibrium.

\subsection{Production Function Estimation}

Cooper and Haltiwanger (1996) discuss evidence on estimates of $\gamma$ from a variety of sources. Cooper and Johri (1997) provide estimates of both contemporaneous and dynamic production complementarities.

A key element in this exercise is, of course, identification. In particular, how can a researcher distinguish variations in output and productivity that arise from a common shock from those that arise endogenously

\footnotetext{
${ }^{31}$ The presence of the complementarity is of interest as a means of propagating and magnifying shocks and also as a basis for multiplicity.

${ }^{32} \mathrm{Of}$ course, there is the issue of which steady state in the event there is more than one. In these papers, there is generally a unique steady state. The multiplicity arises from the presence of a continuum of paths converging to a steady state.
} 
through the production complementarity? The key is to find variables that are independent of the technology shocks $(A)$ but are correlated with output movements. Naturally, one turns to policy interventions for this purpose.

In equilibrium, there is an identification problem that can be seen directly from (15). Imposing $y_{t}=Y_{t}$ and taking logs, (15) becomes:

$$
\ln y_{t}=\frac{\ln A_{t}}{1-\gamma}+\frac{\alpha}{(1-\gamma)} \ln n_{t} \frac{\phi}{(1-\gamma)} \ln k_{t}
$$

Clearly, there is no way to identify the three parameters characterizing the technology from the two regression coefficients.

Baxter and King (1991) estimate $\gamma$ by imposing constant returns to scale $\alpha+\phi=1$. Using demand side instruments, they estimate $\gamma$ of about .33 .

Cooper and Johri (1997) estimate a version of (21) that includes both contemporaneous and dynamic interactions in the production function. Their data is a panel at the two-digit level and they exploit sectoral variation in their estimation. They use monetary innovations as instruments and find evidence of both contemporaneous and dynamic complementarities. In particular, when constant returns to scale to own inputs is imposed, Cooper and Johri (1997) estimate $\gamma=.24$ and a dynamic complementarity of .32.

\subsection{Equilibrium Analysis}

Instead of focusing on the production function parameters alone, one can instead estimate parameters through the equilibrium of the model, as in Farmer and Guo (1995). In some cases, the production complementarity can be large enough that multiple equilibria arise as described in Benhabib and Farmer (1994) and Farmer and Guo (1994). ${ }^{33}$ Here multiplicity arises in the form of multiple paths converging to a locally stable steady state rather than multiple steady states. Given this indeterminacy, sunspots can be used to select the transition path. These economies are of interest as well since they illustrate the estimation of a dynamic sunspot equilibrium.

The economy studied by Farmer and Guo (1995) adds taste shocks to the optimization problem of the individual household in (16). The model thus contains two types of fundamental shocks (tastes and technology) as well as sunspots (effectively Euler equation errors). They reduce the equilibrium analysis to a system of stochastic difference equations and then estimate these using instrumental variables.

Farmer and Guo (1995) find:

- support for the conditions for sunspot equilibria: the labor demand curve is positively sloped and steeper than the labor supply curve.

- Euler equation (demand) shocks are important

- parameter estimates: increasing returns to scale in labor alone though private return not estimated

\footnotetext{
${ }^{33}$ For the analysis of Baxter and King (1991) and Cooper and Johri (1997), the steady state was unique and saddle path stable.
} 
These results seem quite favorable to the sunspots approach. But a couple of points of caution are in order.

First, the model is estimated with errors to the Euler equation. While one interpretation of these errors has to do with the existence of a sunspot equilibrium, Farmer and Guo are appropriately cautious at this point. This shock could in fact reflect a number of sources of variation that included in their structural model, such as variations in government spending and shocks to the intermediation process. ${ }^{34}$ Thus, there is a difficult identification problem that remains in order to complete the argument that fluctuations are driven by confidence alone.

Second, the results on the relative slopes of labor supply (negative) and labor demand (positive) are, to say the least, rather unorthodox. The positively sloped labor demand reflects increasing returns at the social level. ${ }^{35}$ Clearly further evaluation of these results, relative to those found in the labor literature, seems in order.

\section{$5 \quad$ Wage Inequality and Multiple Equilibria}

Labor economics must confront the observation of wage inequality. In the U.S., there is considerable research effort in understanding the earnings inequality between blacks and whites. The possibility, raised in the theoretical work on signalling is that the wage inequality, as well as other difference in labor market experience, may reflect the multiplicity of equilibria rather than fundamental differences between groups.

To see how this may happen, we discuss informally the equilibria outlined in Coate and Loury (1993). Suppose that firms have to assign workers to either a simple or a complex task. Workers prefer to be assigned to the complex task (perhaps reflecting higher wages or more prestige). Firms want to assign only qualified workers to the complex task. Workers become qualified for the complex task by becoming educated (working hard at school) though at a cost that is distributed across the workers (a proxy for ability). The problem is that firms are unable to observe the education of the workers. Instead, they observe a noisy signal of worker qualification. As underlying worker quality is not observable, differences in wages and job assignments will reflect the markets perception of the average ability of workers, given education attainment.

It is then possible to construct equilibria in which workers from one group (call it group A) invest less than workers from another group (B) simply because the perceived returns to education are lower for group A workers. Given this choice by workers in group A, a firm will assign that worker to the complex task iff that worker "scores" higher on a test of qualification than a worker from group B. So, in equilibrium, the returns to education are indeed lower for group A than for group B. Essentially, the fact that few workers in group A decide to acquire human capital pulls down the return to that investment: a firm will interpret a good signal as luck rather than the outcome of training.

These models of job assignment (and even ones with more elaborate structures for wage determination)

\footnotetext{
${ }^{34}$ See Cooper and Ejarque (2000) for a stochastic growth model with intermediation shocks and Ingram, Kocherlakota, and Savin (1994) for maximum likelihood estimates of a stochastic growth model including intermediation and technology shocks.

${ }^{35}$ Whether this is the same as increasing returns at the private level which is often found is not clear since the private returns to labor is not estimated.
} 
can have multiple equilibria. In fact, there is a close link between discrimination and multiplicity brought out in this discussion. If there is discrimination in the sense that otherwise identical groups are treated differently in equilibrium, then there must be multiple equilibria. In the example above, assuming that the fundamentals of the two groups are the same, one could simply switch the labels of the two groups and thus construct an equilibrium in which the returns to education are lower for group B than for group A. ${ }^{36}$

Thus an interesting research question is whether observed time series variation in the black-white wage gap in the U.S. may reflect movements across equilibria rather than changes in fundamentals. Moro (2001) addresses this question.

\subsection{Model}

Here we outline the model used by Moro and then turn to its empirical implementation given the possibility of multiple equilibria. ${ }^{37}$

\section{- Workers}

There are two groups of workers which are identical except for a (pay-off irrelevant) label. For concreteness we call them black and white workers since the empirical evidence is about this form of wage inequality. Workers can either invest in human capital (train) or not. Investment is costly (c) and this cost is distributed across the workers. Further, workers differ in underlying ability. These differences in ability and the cost of investment in human capital are not independent: the cost of training is lower for high ability workers. Workers care about the returns to their labor (the wage) less the cost of training. The wage, as discussed below, reflects this signal of ability. Thus the key to motivating a worker to train is the differential between the wages received in the tasks they may be assigned to.

After making a training decision, workers are tested. These test scores are observable to the firms and along with the workers' group identity are used to determine wages and task assignment.

- Firms

There are many firms in the market. They hire workers and assign them to tasks. Like the Coate and Loury (1993) model, there are two tasks. The first (simple job) does not require any training. The second (complex job) does require training. Firms are unable to observe the ability of workers and are also unable to observe the training of workers. Instead, they observe a signal which reflects the workers training: a worker that trains is more likely to obtain a higher signal. This signal is used by firms to set wages and to determine the task of the worker.

- Equilibrium

Firms hold certain beliefs about the workers and use them to assign workers to tasks and to pay them. The labor market is assumed to be competitive so that wages in the two tasks equal expected marginal

\footnotetext{
${ }^{36}$ Though as discussed by Moro and Norman (2001) these arguments linking multiplicity to discrimination often exploit separability (in production,for example) across the groups of agents.

${ }^{37}$ See Moro and Norman (2001) for a more detailed discussion of a closely related model.
} 
products, conditional on both the signal and the worker type. The equilibrium revolves around the determination of critical test scores that determines the assignment of a worker. If a worker scores in excess of this critical score, then he/she is assigned to the complex task. Else, the worker is assigned to the simple task.

As in the simpler model of Coate and Loury (1993), the key to the multiplicity of equilibria is the implications of prior beliefs for firms' decisions on assignment of workers. If firms believe that the fraction of workers who are training is low, then workers will not perceive any gains to training: good test results will rationally be viewed as good luck. ${ }^{38}$

\subsection{Estimation}

Moro estimates the model using cross sectional data on wages of black and white workers. ${ }^{39}$ The key to the estimation is the mapping from the structural parameters to wage distributions. Then, having observations of wages, the parameters can be estimated.

In a first stage, Moro estimates: the parameters of the testing technology, the cut-offs on the tests and the prior beliefs of firms about the fraction of qualified workers in each group from observations on wage data. He finds (in each of the three cross sections of data)

* the fraction of blacks investing is less than that of whites

* the threshold signal for blacks exceeds that for whites

* the model captures the relative wage distributions quite well

Given these estimates, the second stage estimates the distribution of training costs across the workers as well as the distributions of skills in the two groups. Essentially the identification here is achieved by equilibrium restrictions: construct the distribution of costs such that the observations are part of an equilibrium.

Moro then searches for other equilibria. He concludes that there were multiple equilibria in one of the three years but that the economy selected the equilibrium with lowest differentials in wages and investment.

This is a very different approach to studying multiple equilibria. Effectively, Moro estimates a model assuming that the outcome is from a particular equilibrium and then, through functional forms, searches for other equilibria. One concern with this approach stems from considering this procedure in a setting where there are indeed multiple equilibria. How can we guarantee that an adequate approximation around one of them (assume it is linear!) will necessary find other equilibria?

\section{Social Interactions}

The complementarities that underlie the basic model of Cooper and John (1988) also appear in a growing literature on social interactions. The basic idea is that each agent has a reference group (e.g. a neighbor-

\footnotetext{
${ }^{38}$ Moro (2001) does not supply conditions for multiple equilibria. Moro and Norman (2001) supply intuition and a formal structure.

${ }^{39}$ Importantly, available data on human capital investment and job assignments is not used in the estimation.
} 
hood, a peer group) that influences his/her behavior. For example, the agent may desire to "conform" to the behavior of others and thus will tend to mimic their actions. This creates a strategic complementarity: a single agent has an incentive to mimic the actions of others. ${ }^{40}$ Here though the strategic complementarity is associated with an interaction in preferences rather than in technology (Bryant (1983)) or trading opportunities (Diamond (1982)).

Empirically, there is evidence of correlated behavior across groups of agents. The difficult part of empirical studies is determining whether these correlations reflect common variations or some form of social interaction. This section begins with a review of a basic model and then turns to estimation.

As Manski (1993) states it:

This paper examines the "reflection" problem that arises when a researcher observing the distribution of behavior in a population tries to infer whether the average behavior in some group influences the behavior of the individual that comprise the group.[pg. 532.]

\subsection{Manski's Model}

Suppose that individual behavior is given by:

$$
y=\alpha+\beta E(y \mid x)+z^{\prime} \eta+\mu
$$

where the $y$ denotes an outcome of individual choice (school achievement), $x$ are attributes of an individual's reference group (school peer group), $z$ and $\mu$ are individual attributes where $z$ is observable and $\mu$ is not observed to the researcher.

The individual behavior depends on the group through the term $E(y \mid x) .^{41}$ Thus a key parameter is $\beta$. The determination of the individual choice is part of a social equilibrium as long as $\beta \neq 0$. To solve for the average level of performance given $(x, z)$, use $(22)$ to obtain

$$
E(y \mid x, z)=\alpha+\beta E(y \mid x)+z^{\prime} \eta
$$

Integrating this over $z$, the source of variation across individuals, yields:

$$
E(y \mid x)=\alpha+\beta E(y \mid x)+E\left(z^{\prime} \mid x\right) \eta
$$

Given the assumed linearity in (22), as long as $\beta \neq 1$, there is a unique equilibrium of:

$$
E(y \mid x)=\frac{\alpha}{1-\beta}+E\left(z^{\prime} \mid x\right) \frac{\eta}{1-\beta}
$$

This expression can then be used in (22) yielding:

$$
y=\frac{\alpha}{1-\beta}+E\left(z^{\prime} \mid x\right) \frac{\beta \eta}{1-\beta}+z^{\prime} \eta+\mu
$$

\footnotetext{
${ }^{40}$ Akerlof (1997) presents an informal overview of this approach to social interactions.

${ }^{41}$ It is easiest to think of $x$ here as an indicator variable of group membership so that $E(y \mid x)$ simply measures the average value of $y$ across the members of group $x$.
} 
The social interaction is parameterized by $\beta$ which determines the response of the individual to variations in the mean behavior of the reference group. But, a regression based upon this equilibrium will not necessarily determine all of the parameters uniquely: there is potentially an identification problem. ${ }^{42}$

The intuition parallels that given in the discussion of (21). In that case, the own returns to factor input were impossible to distinguish from production function interactions given aggregate observations. Here, we see that given observations on group and not individual decisions, disentangling social interactions from the individual's response to common sources of variation is not possible. In some cases though, observations on individual choices (again in parallel with the production function estimation) may facilitate identification.

Take, for example, the outcome of students in a high school. Suppose that the average achievement of these students is quite high. Does this reflect the "productive atmosphere" created by the interaction of these students or does it reflect "good luck" of having a great teacher in common? What types of natural experiments and data would allow us to identify the interactions?

Manski (1993) presents certain conditions under which identification is possible in (26). Clearly, if $E\left(z^{\prime} \mid x\right)$ and $\mathrm{z}$ are independent, then the two coefficients obtained from (26) will enable a researcher to distinguish $\beta$ from $\eta$. Otherwise, identification will not be possible. If $E\left(z^{\prime} \mid x\right)$ does not vary across the reference groups, then this term is constant and $\beta$ cannot be estimated even from a cross section of individuals. To appreciate the importance of individual variations, suppose that individuals in a reference group had the same value of $z$. Then again, it is not possible to distinguish $\beta$ from $\eta$.

\subsection{Binary Choice: Brock and Durlauf}

Brock and Durlauf (2000) study related models in which the binary choice of an individual depends on the choices of others, as in the participation complementarities in Cooper (1999). Brock and Durlauf consider the binary choice model:

$$
\max _{\omega_{i} \in\{-1,1\}} V\left(\omega_{i}, Z_{i}, \mu_{i}^{e}\left(\omega_{-i}\right), \epsilon_{i}\left(\omega_{i}\right)\right)
$$

where $\omega_{i}$ is the choice of agent $i, Z_{i}$ is a vector of exogenous variables that influences the preferences of these agent across the alternatives, $\mu_{i}^{e}\left(\omega_{-i}\right)$ represents the beliefs of the agent over the binary choices of others and $\epsilon_{i}\left(\omega_{i}\right)$ is an agent/choice specific shock.

Thus the model of choice is similar to the binary choice models found in other parts of economics with one additional feature: the returns to a particular choice depends on the choices of others. Of course, in this general form the nature of this interaction is not clear. A strategic complementarity is present if the likelihood an agent selects a particular action is higher if "others" select that same action. By "others" we mean agents whose choice influence the payoffs to the agent whose behavior we are studying. As in the

${ }^{42}$ In fact,Manski makes this point even stronger with a more complex model:

$$
y=\alpha+\beta E(y \mid x)+E(z \mid x)^{\prime} \gamma+z^{\prime} \eta+\mu
$$

where $E(\mu \mid x, z)=x^{\prime} \delta$. The shocks $(\mu)$ may be correlated across individuals.

Substituting this equilibrium into (27) implies a reduced form model of:

$$
E(y \mid x, z)=\frac{\alpha}{1-\beta}+E(z \mid x)^{\prime} \frac{\gamma+\beta \eta}{1-\beta}+x^{\prime} \frac{\delta}{1-\beta}+z^{\prime} \eta
$$

Here it is very difficult to identify beta. 
discussion of Manksi's model and in the discussion of the production complementarity model, determining the appropriate reference group is an important open issue in this literature.

Brock and Durlauf (2000) propose specification of utility as:

$$
V\left(\omega_{i}, Z_{i}, \mu_{i}^{e}\left(\omega_{-i}\right), \epsilon_{i}\left(\omega_{i}\right)\right)=u\left(\omega_{i}, Z_{i}\right)-E_{i} \sum_{j \neq i} \frac{J_{i j}}{2}\left(\omega_{i}-\omega_{j}\right)^{2}+\epsilon_{i}\left(\omega_{i}\right)
$$

where $J_{i j}$ is a weight that agent $i$ places on the (expected) choice of $j$. A simple model would have $J_{i j}=J$ iff $j$ is in $i^{\prime} s$ reference group. Then $J$ would be added to the key parameter in estimation of the social interactions effect.

Brock and Durlauf (2000) describe the estimation of this model once structure is placed on the errors (e.g. a logistic specification). Importantly, there is the issue of the treatment of the expectations terms. One approach is to think of these as additional regressors. A second, along the lines explored above, is to supplement the model of choice with a rational expectations restrictions on the expectations of agent $i$. Brock and Durlauf (2000) follow this approach as well though they use the variables that reflect the social interaction as regressors rather than solving for them as part of an equilibrium. ${ }^{43}$

Not surprisingly, many of the same identification problems arise in the binary choice framework as in Manski's model. However, Brock and Durlauf (2000) argue that variations in the equilibrium selected across "neighborhoods" can provide a basis of identification.

\section{Conclusion}

This paper began with the often expressed concern that models build upon multiple equilibria, despite their intuitive appeal, were incapable of confronting data. The point of this paper was to argue, through selected examples, that in fact progress has been made to use observations to estimate (some) parameters of these models and to falsify these models.

Note that the empirical approach described here rests upon versions of simulated method of moments and/or maximum likelihood. This emphasis does not imply that alternative means of model evaluation should not be considered. For example, there may be certain experiments initiated by government action (the introduction of deposit insurance, aspects of the National Industrial Recovery Act, as in Cooper and Haltiwanger (1993)) where the response of the economy reveals the resolution of strategic uncertainty.

Of course, a paper of this type must miss some important aspects of the ongoing research on this topic. Unfortunately, the paper lacks any discussion of work on experimental coordination games. In that research, the dynamics of learning through the interaction of player is quite rich and may ultimately provide the basis for the empirical evaluation of models of learning and selection. Further, recent results on coordination games with private information, are unfortunately excluded for consideration.

\footnotetext{
${ }^{43}$ In the discussion of the Manski model we solved for the equilibrium values of the groups means and then substituted them back into the agents choice. The same issues arise in the estimation of production functions. Using plant level data, for example, one can use sectoral or aggregate output as a regressor and with appropriate instruments obtain consistent estimates.
} 


\section{References}

AiYagari, R. (1995): "Comments on Farmer and Guo's" The econometrics of Indeterminacy: An Applied Study"," Federal Reserve Bank of Minneapolis, Staff Report 196.

Akerlof, G. (1997): “Social Distance and Social Decisions,” Econometrica, 65(5), 1005-1027.

Azariadis, C. (1981): "Self-Fulfilling Prophecies," Journal of Economic Theory, 25, 380-96.

Azariadis, C., And R. Guesnerie (1986): "Sunspots and Cycles," Review of Economic Studies, 53(5), $725-37$.

Baxter, M., And R. King (1991): "Production Externalities and Business Cycles," Federal Reserve Bank of Minneapolis, Discussion Paper 53.

Benhabib, J., and R. Farmer (1994): "Indeterminacy and Increasing Returns," Journal of Economic Theory, 63, 19-41.

Bisin, A. Moro, A., And G.Topa (2002): "The Empirical Content of Models with Multiple Equilibria," mimeo, New York University.

Breshnahan, T., And P. Reiss (1991): "Empirical Models of Discrete Games," Journal of Econometrics, $64,57-81$.

Brock, W., and S. Durlauf (2000): "Interactions Based Models," NBER, Technical Working Paper no. 258.

Bryant, J. (1983): "A Simple Rational Expectations Keynes-Type Model," Quarterly Journal of Economics, 97, 525-29.

(1987): "The Paradox of Thrift, Liquidity Preference and Animal Spirits," Econometrica, 55, $1231-36$.

Carlsson, H., and E. van Damme (1993): "Global Games and Economic Selection,” Eca, 61, 989-1018.

CoAte, S., And G. Loury (1993): "Will Affirmative Action Policies Eliminate Negative Stereotypes?," American Economic Review, 83(5), 1220-1240.

Cooper, R. (1999): Coordination Games: Complementarities and Macroeconomics. Cambridge University Press.

Cooper, R., and D. Corbae (2001): "Financial Collapse and Active Monetary Policy: A Lesson from the Great Depression," Federal Reserve Bank of Minneapolis: Staff report 289, forthcoming, Journal of Economic Theory.

Cooper, R., and J. Ejarque (2000): "Financial Intermediation and Aggregate Fluctuations: A Quantitative Analysis," Macroeconomic Dynamics, 4, 423-447. 
Cooper, R., And J. Haltiwanger (1993): "Autos and the National Industrial Recovery Act: Evidence on Industry Complementarities," QJE, 108, 1043-72.

(1996): "Evidence on Macroeconomic Complementarities," The Review of Economics and Statistics, $77,78-93$.

Cooper, R., And A. John (1988): "Coordinating Coordination Failures in Keynesian Models," Quarterly Journal of Economics, 103, 441-63.

Cooper, R., And A. Johri (1997): "Dynamic Complementarities: A Quantitative Analysis," Journal of Monetary Economics, 40, 97-119.

Diamond, P. (1982): "Aggregate Demand management in search equilibrium," Journal of Political Economy, 90(5), 881-894.

Farmer, R., and J. T. Guo (1994): "Real Business Cycles and the Animal Spirits Hypothesis," Journal of Economic Theory, 63, 42-72.

(1995): "The econometrics of Indeterminacy: An Applied Study," Carnegie Rochester Series on Public Policy, 42.

Gourieroux, C., And A. Monfort (1996): Simulation-Based Econometric Methods. Oxford University Press.

Hansen, L. P. (1982): "Large Sample Properties of Generelized Method of Moments Estimators," Econometrica, 50, 1029-1054.

Ingram, B., N. Kocherlakota, and N. Savin (1994): "Explaining Business Cycles:A multiple-shock aproach," JME, 34, 415-28.

Jovanovic, B. (1989): "Observable Implications of models with Multiple Equilibria," Econometrica, 57, $1431-38$.

Manski, C. (1993): "Identification of Endogenous Social Effects: The Reflection Problem," Review of Economic Studies, 60(3), 531-42.

Moro, A. (2001): "The Effects of Statistical Discrimination on Balck-White Wage Inequality: Estimating a Model with Multiple Equilibria," mimeo.

Moro, A., And P. Norman (2001): "A General Equilibrium Model of Statistical Discrimination," mimeo.

Weil, P. (1987): "Increasing Returns and Animal Spirits," American Economic Review, 79, 889-94.

Woodford, M. (1987): "Three Questions about sunspot equilibria as an Explanation of Economic Fluctuations," American Economic Review, 77(2), 93-98. 